\title{
Alterações físicas, emocionais e psicossociais de idoso na pandemia por coronavírus
}

\author{
Physical, emotional and psychosocial changes in the elderly in the coronavirus pandemic \\ Cambio físicos, emocionais y psicosociales em los ancianos em La pandemia de coronavirus
}

Recebido: 10/05/2021 | Revisado: 16/05/2021 | Aceito: 18/05/2021 | Publicado: 06/06/2021

\author{
Ana Maria Carneiro Oliveira \\ ORCID: https://orcid.org/0000-0002-5158-921X \\ Centro Universitário Santo Agostinho, Brasil \\ E-mail:anamariacarneirooliveira20@gmail.com \\ Erica da Silva Sousa \\ ORCID: https://orcid.org/0000-0002-9426-9216 \\ Centro Universitário Santo Agostinho, Brasil \\ E-mail: ericassilva2014@icloud.com \\ Disraeli Reis da Rocha Filho \\ ORCID: https://orcid.org/0000-0002-6691-5866 \\ Centro Universitário Santo Agostinho, Brasil \\ E-mail: disraelirocha@gmail.com
}

\begin{abstract}
Resumo
Relacionar o isolamento social no idoso e mortalidade, e seus efeitos, psíquicos, emocionais e sociais, em idosos, causados pela pandemia da COVID-19. Pesquisa realizada como Revisão Bibliográfica da Literatura pelo método de revisão integrativa, que resume a literatura teórica para fornecer compreensão ampla sobre um dado fenômeno. A estratégia PICo, foi utilizada para construir questões de pesquisa. O grupo populacional mais vulnerável e que apresenta maior letalidade em relação à COVID-19 são os idosos. Esse fato deve-se a imunossenescência, que aumenta a vulnerabilidade às doenças infectocontagiosas e traz os piores prognósticos para aqueles com doenças crônicas não transmissíveis. No entanto, o ser humano não é somente biofísico, mas também psicossociocultural. Cabe aos profissionais de saúde, sugerir medidas para conter os danos à saúde do idoso, como estratégias de orientações sobre o isolamento social para evitar a contaminação, portanto, necessitam de conhecimentos específicos sobre essa população, voltados não somente para o combate do COVID-19, bem como dos demais problemas de saúde que afeta essa população.
\end{abstract}

Palavras-chave: COVID-19; Idosos; Isolamento social.

\begin{abstract}
s
To relate social isolation in the elderly and mortality, and its effects, psychic, emotional and social, in the elderly, caused by the pandemic of COVID-19. Research conducted as a Bibliographic Literature Review by the integrative review method, which summarizes theoretical literature to provide broad understanding about a given phenomenon. The PICo strategy, was used to construct research questions. The most vulnerable population group, with the highest lethality in relation to COVID-19, is the elderly. This fact is due to immunosenescence, which increases vulnerability to infectious diseases and brings the worst prognosis for those with chronic non-communicable diseases. However, the human being is not only biophysical, but also psychosociocultural. It is up to health professionals to suggest measures to contain the damage to the health of the elderly, as strategies for guidance on social isolation to avoid contamination, therefore, need specific knowledge about this population, aimed not only to combat COVID-19, as well as other health problems affecting this population.
\end{abstract}

Keywords: COVID-19; Elderly; Social isolation.

\section{Resumen}

Relacionar el aislamiento social en los ancianos y la mortalidad, y sus efectos, psíquicos, emocionales y sociales, en los ancianos, causados por la pandemia de COVID-19. Investigación realizada como Revisión de la Literatura Bibliográfica mediante el método de revisión integradora, que resume la literatura teórica para proporcionar una amplia comprensión sobre un fenómeno determinado. La estrategia PICo, se utilizó para construir las preguntas de investigación. El grupo de población más vulnerable y con mayor letalidad en relación con el COVID-19 es el de las personas mayores. Este hecho se debe a la inmunosenescencia, que aumenta la vulnerabilidad a las enfermedades infecciosas y conlleva el peor pronóstico para los enfermos crónicos no transmisibles. Sin embargo, el ser humano no es sólo biofísico, sino también psicosociocultural. Corresponde a los profesionales de la salud sugerir medidas para contener el daño a la salud de los ancianos, como las estrategias de orientación sobre el aislamiento social para evitar la contaminación, por lo tanto, necesitan un conocimiento específico sobre esta población, dirigido no sólo a combatir el COVID-19, así como otros problemas de salud que afectan a esta población.

Palabras clave: COVID-19; Personas mayores; Aislamiento social. 


\section{Introdução}

O novo CoronaVírus designado como Severe Acute Respiratory Syndrome Corona Vírus-2 (Sars-Cov-2), e como Corona Vírus Disease-19 (COVID-19) para a doença, promove infecção aguda, não há estado crônico de infecção e os seres humanos não são seus hospedeiros naturais. O coronavírus humanos afetam principalmente no trato respiratório e causam infecções que variam de resfriados comuns a síndrome respiratória aguda grave (Zhang, 2020).

Em Wuhan, na China, foi diagnosticado o primeiro caso de Coronavírus, e em 11 de março, declarada Pandemia Mundial para a Organização Mundial de Saúde. No Brasil, o primeiro caso positivo foi anunciado em 26 de fevereiro de 2020 , sendo um homem morador de São Paulo, de 61 anos, que esteve na Itália. O primeiro óbito brasileiro confirmado ocorreu em 17 de março de 2020, com um idoso de 62 anos, que tinha diabetes e hipertensão que são comorbidades. Então, os idosos estão no centro da discussão da pandemia COVID-19 (Secretaria de Vigilância em Saúde, 2019)

A população mais vulnerável para adquirir o COVID-19 são os idosos, pelo fato de serem do grupo de risco. Este fato deve-se a imunossenescência, que aumenta a vulnerabilidade às doenças infectocontagiosas e os piores prognósticos para aqueles com doenças crônicas. Porém o ser humano não é somente biofísico, envolvendo aspectos psicossociocultural. Neste contexto, alterações físicas, emocionais e psicossociais de idosos se constituem um tema interessante para a realização desta revisão, por ser ao mesmo tempo, um problema de saúde na realidade mundial, pois se trata de uma pandemia, "a COVID-19", (Vman, $\mathrm{N}$ et al.; 2020).

Os idosos possuem suas próprias peculiaridades, além da sua diversidade do envelhecimento humano. Nesse cenário, apesar dos conceitos fundamentais da epidemiologia, não se pode abster dos fundamentos da teoria e prática da gerontologia. A citar o distanciamento social, são adotadas medidas para evitar a propagação da COVID-19. A gerontologia visando minimizar seus efeitos evitando o isolamento social considerado como síndrome geriátrica, e o medo da morte pois esses idosos ficam mais vulneráveis a qualquer tipo de situação desconfortante (Hugues, Mcmurd \& Gutrie, 2020).

O isolamento social é feito por um período de 14 dias, tempo em que o vírus leva pra se manifestar no corpo. Diante de um cenário de pandemia pessoas tiveram que parar a sua vida, domino trabalho e viver um período de isolamento. Portanto os idosos tornaram-se o centro de associação da doença COVID-19 ao elevado risco de morte, por ser um dos grupos de risco. Além de se tornar alvo de "perseguição" para a maioria da população no sentido de "superproteger" e forçando uma situação de isolamento social (Santos \& Rodrigues, 2020).

A pandemia trouxe consigo vários fatores que podem que podem influenciar na condição de saúde mental, o excesso de informação, do distanciamento social, e suas repercussões na empregabilidade e sustento de muitos. Podem o ocorrer impacto como dano econômico, e perda de entes queridos. Essas situações podem favorecer agravos ou surgimentos relacionados à saúde mental. Por isso sempre devemos estar mais próximos dos idosos, para passar uma confiança, e uma sensação de superproteção O grupo de pessoas está mais vulneráveis e com maior apresentação de maior letalidade com relação ao COVID-19 são os idosos, pelo fato de terem maiores comorbidades (Andrade \& Correia, 2019).

Supõe-se que em todo o mundo idosos estejam muito prejudicados nesse período de pandemia da COVID-19. Pelo fato do isolamento social, eles se sentem mais solitários e submetidos a vários tipos de sofrimento e restrições, especialmente nessa faixa etária onde carecem de muito afeto e cuidados de seus familiares. Acredita-se que muitos estudos já tenham sido realizados sobre estas alterações, mas pouco se conhece a respeito das evidências apontadas nesses trabalhos que tratam desses prejuízos, considerando ser esta pandemia muito recente. Assim, estabeleceu-se como questão norteadora desse estudo: Qual a produção dos profissionais de saúde/enfermagem sobre os efeitos físicos, emocionais e psicossociais da pandemia da COVID19 em idosos? 


\section{Metodologia}

Literatura teórica para fornecer compreensão ampla sobre um dado fenômeno. Desta forma, a RI tem potencial para construir ciência de enfermagem, transformando pesquisas, práticas e iniciativa políticas já conhecidas. Esta RI percorre as seguintes etapas: formulação da questão norteadora busca eletrônica por publicações, seleção dos dados, análise, interpretação dos dados e apresentação dos resultados (Santos et al., 2005).

Os dados extraídos dos artigos para esta revisão integrativa foram escolhidos de forma a atender os interesses dos pesquisadores, atendendo a questão da pesquisa e os descritores ou palavras-chaves utilizadas. A análise foi feita em etapas: Na primeira etapa de análise, se buscará a condução da revisão, de forma criteriosa, sendo que a objetividade inicial predisporá todo o processo para uma análise direcionada e completa, com conclusões de fácil identificação e aplicabilidade. A questão norteadora da revisão integrativa pode ser, por exemplo, uma intervenção específica, ou mais abrangente, examinando diversas intervenções ou práticas na área da saúde ou de enfermagem (Gonong et al., 2005).

$\mathrm{Na}$ segunda etapa, que está ligada à anterior, levando em conta a abrangência do assunto a ser estudado que determinará o procedimento de amostragem. Os revisores (pesquisadores) devem refletir sobre este ponto, adotando procedimento de inclusão e exclusão dos conteúdos dos artigos de forma a conduzir-se criteriosamente e de maneira transparente, uma vez que a representatividade de amostra é um indicador de profundidade. A melhor estratégia seria colocar todos os estudos, e pesquisar padrões de possíveis influências metodológicas nos resultados (Whittemore, 2005).

Na quinta etapa, são interpretados dando origem aos resultados. Essa etapa diz respeito à discussão dos principais resultados na pesquisa convencional. A identificação de lacunas permite que o revisor aponte sugestões pertinentes para futuras pesquisas direcionadas para a melhoria da assistência à saúde. Na sexta etapa, será a apresentação da revisão/síntese do conhecimento. A maior dificuldade para delimitar as conclusões obtidas da revisão é o quanto nem todas as características e os resultados dos estudos foram relatados nas fases anteriores pelo revisor (pesquisador). Esta etapa consiste na elaboração do documento que deve contemplar a descrição das etapas percorridas pelo revisor e os principais resultados evidenciados da análise dos artigos incluídos. (Roman Friedlnder \& Ganong, 1998)

Os artigos para o estudo foram selecionados nas bases: Scielo, Lilacs, Medlane e Pubmed, pelos os descritores aplicados seguindo os Descritores em Ciência da Saúde (DeCS) e o Medical Subject Heading (MeSH): "Coronavírus" (Coronavírus), "Idosos", (Elderly), "Pandemia", (pandemic).

A estratégia PICo (População, Interesse e Contexto) foi utilizada para construir questões de pesquisa abordada na parte introdutória deste estudo: Qual a produção dos profissionais de saúde/enfermagem sobre os efeitos físico, e emocionais, psicossociais da pandemia da COVID-19, em idosos? Esta reuniu estudos de naturezas diversas, oriundos da clínica, do gerenciamento de recursos humanos e materiais, da busca de instrumentos para avaliação de sintomas entre outras. A pergunta de pesquisa adequada (bem construída) possibilita a definição correta de que informações (evidências) são necessárias para a resolução da questão clínica de pesquisa, maximiza a recuperação de evidências nas bases de dados, foca o escopo da pesquisa e realização de busca desnecessária (Akobeng et al., 1999).

Os artigos para a pesquisa foram filtrados pelo critério de inclusão: artigos completos, publicados nos últimos 5 anos, em português, espanhol e inglês; e que seguem os descritores e o objeto de estudo da pesquisa. Como critérios de exclusão, foram eliminados: os estudos de revisão, dissertações, teses, editoriais, carta ao editor, relatos de experiência, resumos de eventos, disponíveis na íntegra, artigos que não tratavam do tema proposto, teses, capítulos de livros e editoriais, os repetidos (duplicados), publicados fora do período selecionado, que não estão de acordo com as línguas selecionadas, com os descritores e objeto de estudo. (Figura 1). 
Figura 1. Registros de artigos encontrados nas bases de dados Lilacs, Scielo, Medline e Pubmed.

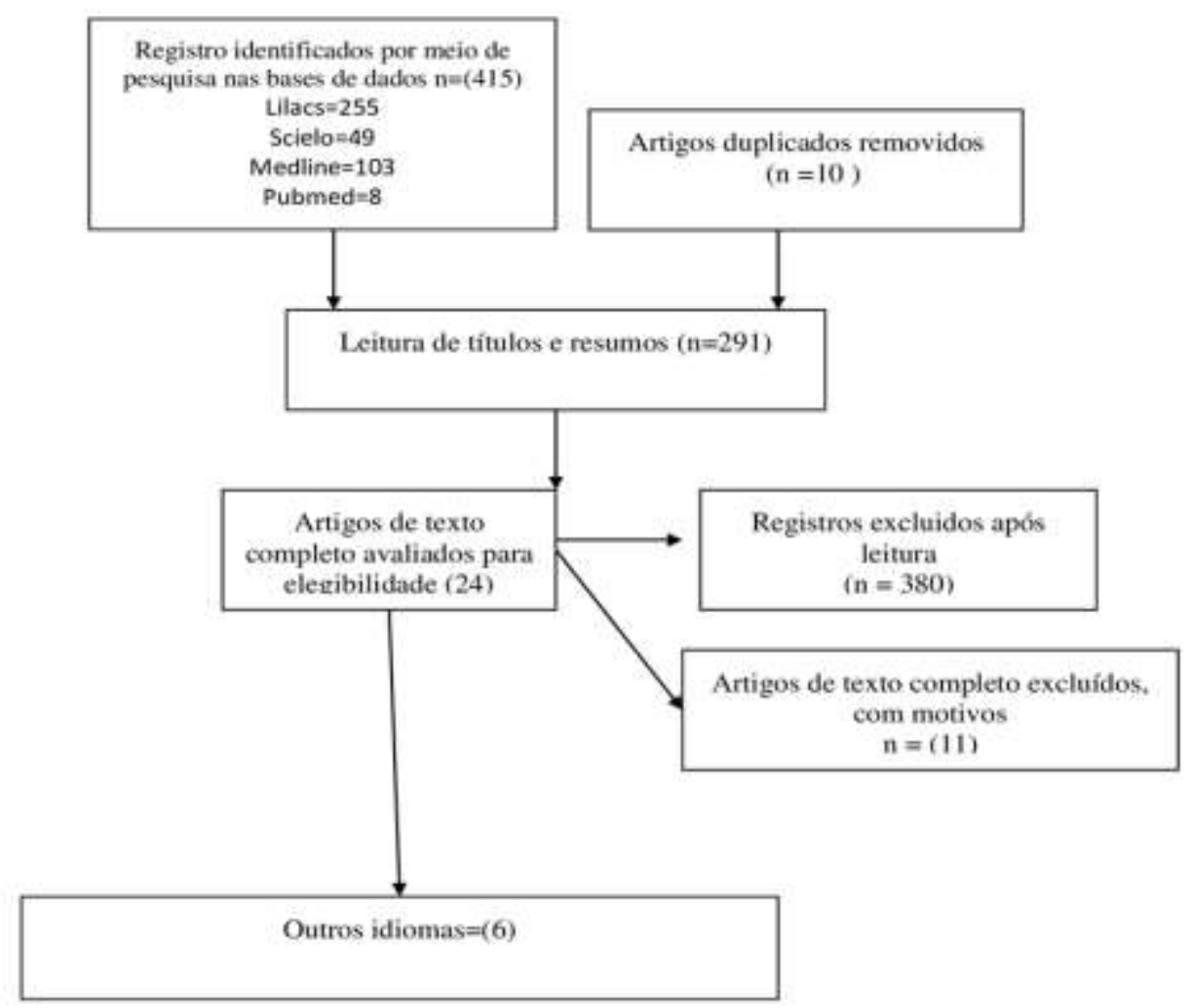

Fonte: Moher D, Liberati A, Tetzlaff J, Altman DG, The PRISMA Group (2009).

\section{Resultados}

Para ZHANG o grupo populacional mais vulnerável e que apresenta maior letalidade em relação à COVID-19 são os idosos, principalmente aqueles com comorbidades, seja ela hipertensão arterial, ou diabetes. Esse fato deve-se a imunossenescência, que aumenta a vulnerabilidade às doenças infectocontagiosas e traz os piores prognósticos para aqueles com doenças crônicas não transmissíveis. No entanto, o ser humano não é somente biofísico, mas também psicossociocultural.

Para Organização Mundial da Saúde (OMS), 2020 consolidou um total de 15.538.736 casos confirmados e 634.325 óbitos em decorrência da doença em 216 países com idades maiores. Estados Unidos, Brasil, Índia, Rússia, África do Sul e Peru são os países com o maior quantitativo de casos até o momento a grande maioria são idosos com comorbidades, atingindo principalmente idosos acima de 80 anos (Figura 2).

Figura 2. Taxa de mortalidade do COVID-19 por idade.

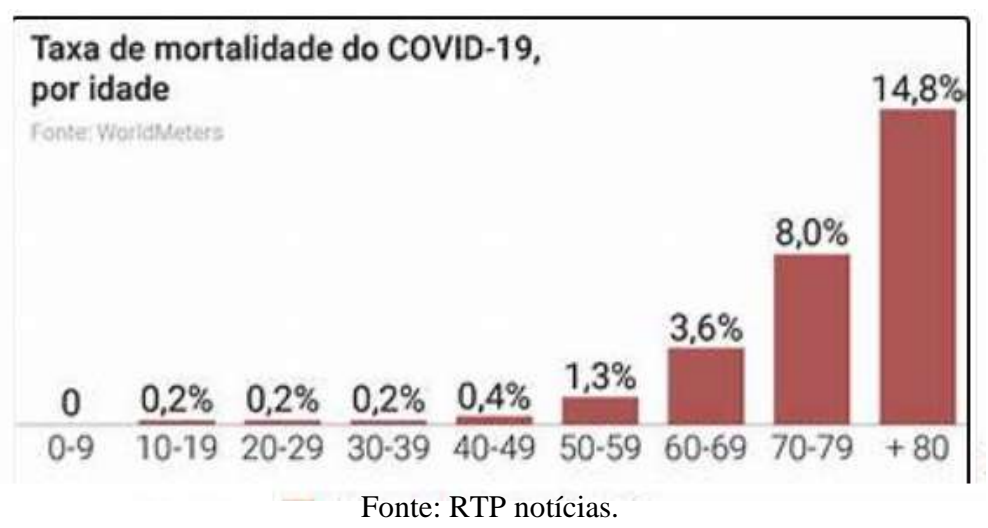


Para o Ministério da Saúde, 2020, em meio às orientações, diretrizes e decretos com recomendações para que os idosos permanecessem em distanciamento social, a heterogeneidade dos idosos brasileiros evidencia as dificuldades em atender a suas demandas, expondo fragilidades, principalmente familiares e de rede de apoio. Porém, não se pode decidir o destino do planeta isoladamente.

Para Morais, et al, apesar de extremamente relevante para a redução da transmissão da doença e conseqüentemente do número de casos e óbitos, tal distanciamento, em médio e longo prazo, também traz sérios prejuízos para a atividade econômica em todos os seus níveis e para a vida em sociedade. Apesar de extremamente relevante para a redução da transmissão da doença e conseqüentemente do número de casos e óbitos, tal distanciamento, em médio e longo prazo, também traz sérios prejuízos para a atividade econômica em todos os seus níveis e para a vida em sociedade (Figura 3).

Figura 3. Índice de isolamento social.

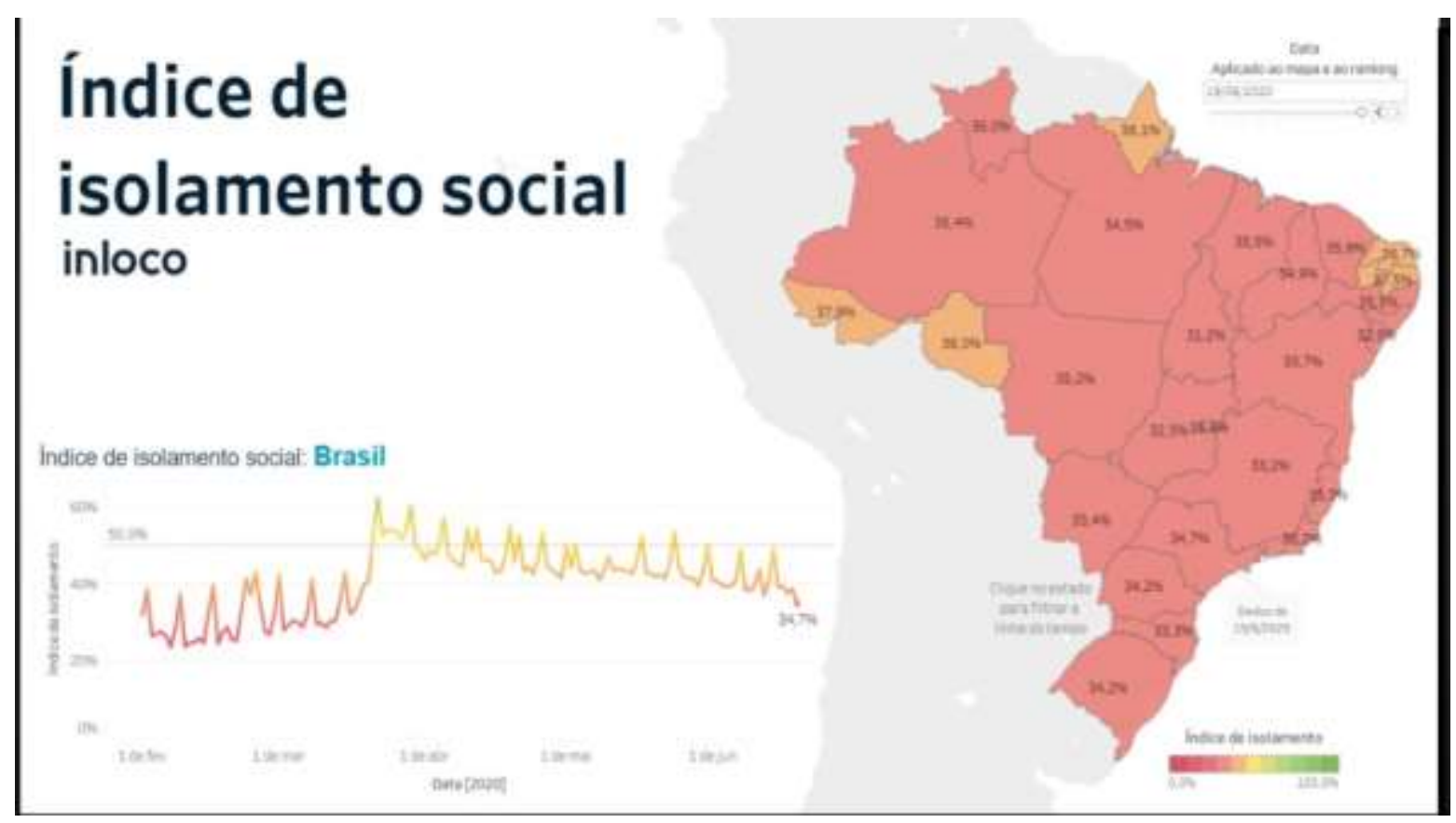

Fonte: Coepbrasil.

Para Brasil, Sociedade Brasileira de Geriatria e Gerontologia (SBGG) Todos os idosos, especialmente portadores de comorbidades como diabetes, hipertensão arterial, doenças do coração, pulmão e rins, doenças neurológicas, em tratamento para câncer, portadores de imunossupressão entre outras, e aqueles com mais de 80 anos e portadores de síndrome de fragilidade, necessitam adotar medidas de restrição de contato social.

Para Zhang, apesar da evolução clínica não ser clara, estudos têm demonstrado relação direta e importante da faixa etária do paciente e a sua carga de morbidades (número e gravidade) com o aumento do risco para a incidência de desfechos clínicos desfavoráveis, com um número de mortes altas, tais como internação hospitalar, necessidade de unidade de tratamento intensivo (UTI) (Tabela 1). 
Tabela 1. Faixa etária do paciente e sua carga de morbidade.

\begin{tabular}{|c|c|c|}
\hline CHA2DS2-VASC & Descriçao & Pontos \\
\hline c & Insuficiência cardiaca & 1 \\
\hline $\mathrm{H}$ & Hipertensco & 1 \\
\hline$A_{2}$ & Idade ( 275 anos) & 2 \\
\hline D & Diabetes mellitus & 1 \\
\hline $\mathbf{s}_{2}$ & AIT ou AvC prévio & 2 \\
\hline v & Doença vascular (IAM prévia, placa ab́rtica, doença arterial periférica) & 1 \\
\hline A & Idade (65-74 anos) & 1 \\
\hline c19 & Suspeita ou confirmaçao de COVID-19 & 1 \\
\hline
\end{tabular}

Fonte: Sociedade Brasileira de Angiologia e de cirurgia vascular.

Para Brasil Ministério da Saúde, 2020. Os dados do último boletim do Ministério da Saúde, datado de 04 de abril de 2020, revelaram 10.278 casos e 431 mortes, sendo que mais de 80\% dos óbitos pelo novo Coronavírus foram de pessoas idosas e que quase o mesmo montante apresentava pelo menos um dos fatores de risco constantemente relacionados à Covid-19, com destaque para as cardiopatias e diabetes. Isso nos faz refletir que medidas mais abrangentes sejam necessárias para proteger essa população.

Segundo Comas Herrera et al, dos 240.287 óbitos por COVID-19 considerados, ocorreria em instituições de longa permanência para idosos um total de 107.528 mortes em idosos nestas instituições (intervalo de 95,0\% de confiança de 107.269 a 107.788). Esses óbitos perfazem 61,9\% de todos os 175.435 óbitos por COVID-19 em idosos. A Região Sudeste tem a maior parte dos óbitos do Brasil, equivalendo a 48.779 mortes (intervalo de 95,0\% de confiança de 48.661 a 48.898 ), seguida de Região Nordeste, que teve 28.451 mortes (intervalo de 95,0\% de confiança de 28.382 a 28.520. Como mostra na Tabela 2

Tabela 2. Óbitos no Brasil.

\begin{tabular}{l||l|l}
\hline \multicolumn{1}{c||}{ região: } & confirmados: & óbitos: \\
\hline \hline Norte & 527 & 16 \\
\hline \hline Nordeste & 1.642 & 59 \\
\hline \hline Sudeste & 6.295 & 329 \\
\hline \hline Centro- & 675 & 10 \\
Oeste & & 17 \\
\hline \hline Sul & 1.139 & $\begin{array}{l}431(4,2 \% \text { de } \\
\text { mortalidade', }\end{array}$ \\
\hline \hline TOTAL & 10.278 & \\
\hline
\end{tabular}

Fonte: Conexão verdade. 
De acordo com o Caderno de Saúde publica o total de idosos aposentados que cumpria isolamento social é de 30,9\%, sendo 20,2\% para o sexo masculino no distanciamento intenso. Já os que não cumpriam o distanciamento (saiu para mercado, compras, etc.) do total de idosos 56,9\%, para o se sendo sexo masculino e $61,0 \%$ e para o sexo feminino 54,0\%. Não fez distanciamento ou pouco alterou sua rotina total de idosos 12,2\%, sendo do sexo masculino $19 \%$ e feminino 7,0\%.

De acordo Klinenberg, idosos aposentados ou que já não trabalhavam antes da pandemia apresentaram maior adesão às medidas de distanciamento social total (40,4\%; IC95\%: 34,8; 46,3 e 41,7\%; IC95\%: 33,8; 50,0). Cerca de 10\% dos idosos que continuaram trabalhando em home office ainda assim não aderiram ao distanciamento (IC95\%: 5,4; 17,4). Dos que exerceram alguma atividade essencial durante a pandemia, 44,2\% (IC95\%: 33,9; 55,1) não aderiram ao distanciamento social. De acordo com a Tabela 3.

Tabela 3. Distribuição (\%) da situação socioeconômica, do domicílio e de trabalho dos idosos segundo a adesão ao distanciamento social durante a pandemia de COVID-19. Brasil, 2020.

\begin{tabular}{|c|c|c|c|c|c|c|c|}
\hline \multirow[t]{2}{*}{$\begin{array}{l}\text { Situação socioeconômica e } \\
\text { demográfica }\end{array}$} & \multicolumn{2}{|c|}{ Distanciamento total } & \multicolumn{2}{|c|}{$\begin{array}{c}\text { Distanciamento } \\
\text { intenso (saiu para } \\
\text { mercado, compras etc.) }\end{array}$} & \multicolumn{2}{|c|}{$\begin{array}{l}\text { Não fez distanciamento ou } \\
\text { pouco alterou sua rotina }\end{array}$} & \multirow[t]{2}{*}{ Valor de $p$} \\
\hline & $\%$ & IC95\% & $\%$ & IC $95 \%$ & $\%$ & IC95\% & \\
\hline Total de idosos $(n=9.102)$ & 30,9 & 27,$8 ; 34,1$ & 56,9 & 53,$5 ; 60,3$ & 12,2 & 10,$1 ; 14,7$ & - \\
\hline Sexo & & & & & & & $<0,001$ \\
\hline Masculino & 20,2 & 15,$6 ; 25,8$ & 61,0 & 54,$7 ; 66,5$ & 19,0 & 14,$7 ; 24,2$ & \\
\hline Feminino & 39,1 & 35,$3 ; 42,9$ & 54,0 & 50,$0 ; 57,9$ & 7,0 & 5,$4 ; 8,8$ & \\
\hline
\end{tabular}

Fonte: ConVid - Pesquisa de Comportamentos.

\section{Discussão}

A disseminação do coronavírus se dá por gotículas de saliva, espirro, tosse, catarro, contato, contato pessoal com toque ou aperto de mão, contato com objetos ou superfícies contaminadas, acompanhando de contato com a boca, nariz ou olhos. Por isso a importância de manter esses idosos em distanciamento social. É importante que os familiares desses idosos incentivem a prática de atividade física dentro de casa, algo que possa ocupar esse espaço vazio que o falta (Brasil Ministério da saúde, 2020).

A pandemia expôs a fragilidade e particularidades de populações especialmente vulneráveis, como a dos idosos. Logo ao mesmo tempo trouxe diversos impactos na vida social, alterações físicas, mentais e psicossociais. É importante que cuidadores e familiares fiquem atentos aos primeiros sinais de tristeza, desânimo, falta de energia, ou ocasionando febre persistente. A morte decorrente da COVID -19 aumenta com a idade do indivíduo e é mais freqüente em pessoas com mais de 60 anos, especialmente aquelas com condições crônicas de saúde por serem mais vulneráveis (Brasil, Sociedade Brasileira de Geriatria e Gerontologia, 2020).

Para a terceira idade uma pandemia, é considerado negativamente um momento de grandes transformações para o indivíduo principalmente para a pessoa idosa. Isso inclui uma série de mudanças físicas, psicossociais, emocionais, doenças, afastamento do trabalho ou perda de pessoas queridas, além de uma redução da independência e autonomia de modo geral. A depressão é consequiências de vários fatores que mais acometem os idosos durante o período de pandemia. Em meio a medidas de precaução como o isolamento social e a redução dos números de óbitos, são muitas informações circulando, algumas verdadeiras e outras falsas assim acabam sendo mais um impacto negativo para o idoso (Clafield. et al., 2020).

Durante a pandemia por Coronavírus em que os idosos devem permanecer em isolamento social, é algo importante quando se fala no contexto em saúde. Devido ao grande número de incidência causado pela COVID-19, alguns países têm 
adotado medidas de controle como da o isolamento social de escolas, creches, idosos, pessoas com comorbidades a quarentena de toda a população. O índice de isolamento social no Brasil não é tão satisfatório para assim manter as medidas de segurança (Brooks, et al., 2020)

\section{Conclusão}

Altas letalidades por SARS-CoV-2 têm sido, em sua maior parte, associadas a pacientes idosos ou à presença de comorbidades mais comuns nestes pacientes, sendo superiores a um quinto dos acometidos com mais de 80 anos. As restrições impostas pelo isolamento social implicam no desenvolvimento saudável desses idosos, nos aspectos físicos, emocionais e psicossociais.

Os idosos requerem mais atenção no período de pandemia, por serem do grupo de risco, e podem apresentar COVID19 nas formas mais avançadas, por em geral apresentarem doenças como hipertensão, diabetes, situações de imunossupressão, sendo assim necessárias medidas de precauções, promoção e prevenção da saúde, como programas adequados e direcionados para este público. Deste modo, cabe aos profissionais de saúde sugerir medidas para conter os danos, como estratégias de orientações sobre o isolamento social para evitar a contaminação. Portanto, necessitam de conhecimentos específicos sobre essa população voltada não somente para o combate do COVID-19, bem como dos demais problemas de saúde que afeta essa população.

A COVID-19, o distanciamento social e pandemia trouxeram várias consequências para o publico idoso, entre elas a mentalidade comprometida, fragilidade com relação a saúde, e até mesmo a violência contra os mesmos. Este público deve ser visto mais de perto, por motivos relacionados à melhora da qualidade de vida de forma efetiva da população idosa no momento pós-pandemia. São necessários maiores investimentos em cuidados e serviços como forma de prevenção para garantir segurança e minimizar os riscos causados, mais estudos que tratem a respeito dos impactos da pandemia nos idosos, visto que ainda estamos no período de pandemia.

\section{Referências}

Alex, N. A., \& Isabella, M. O. M. (2020). Implicações na saúde mental de idosos diante do contexto pandêmico da COVID-19. Revista enfermagem atual. Almeida, C. F. et al (2020). COVID-19: seus impactos clínicos e psicológicos na população idosa. Brazilian Journal of Development, 6(7), $49811-49824$.

Araújo, B, C. G. B \& Saretto, C, B. (2021). O impacto da pandemia COVID-19 sobre a fragilidade física e a capacidade funcional de idosos. Revista FisiSenectus.

Almeida, H., Karina, \& Karina, R. F. (2020). Saúde do idoso em tempos de pandemia COVID-19. Cogitare Enfermagem, 25.

Barbosa, I. R. et al. (2020). Incidência e mortalidade por COVID-19 na população idosa brasileira e sua relação com indicadores contextuais: um estudo ecológico. Revista Brasileira de Geriatria e Gerontologia, 23(1).

Karina, S. A. H., Lisiane, C. P. B. \& Anderson, A. C. (2020). Caminho da esperança nas relações envolvendo os idosos: Olhar da complexidade sob pandemia do COVID-19 Texto e contexto enfermagem.

Leão, L. R. B., Ferreira, V. H. S., \& Faustino, A. M.(2020). O idoso e a pandemia do Covid-19: uma análise de artigos publicados em jornais. Brazilian Journal of Development, 6(7), 45123-45142.

Lima, B. P. C., Lima, L. C. R. \& Dantas, S. C.(2020). Pandemia da covid-19 e idosos como população de risco: aspectos para educação em saúde. Cogitare Enfermagem, v. 25.

Lima, K. C. (2020). A pessoa idosa domiciliada sob distanciamento social: possibilidades de enfrentamento à covid-19. Rev. Bras. Geriatr. Gerontol.

Lima, S. O et al., (2020). Impactos no comportamento e na saúde mental de grupos vulneráveis em época de enfrentamento da infecção COVID-19: Revisão narrativa. Revista Eletrônica Acervo Saúde, (46), e4006-e4006.

Hammerchmidt, \& Santana, R. F.(2020). Saúde do idoso em tempos de pandemia COVID-19 Cogitare enferm.

Machado, C. J et al., (2020). Estimativas de impacto da COVID-19 na mortalidade de idosos institucionalizados no Brasil. Departamento de Medicina Preventiva e Social, Faculdade de Medicina, Universidade Federal de Minas Gerais (UFMG) 
Research, Society and Development, v. 10, n. 6, e44310615964, 2021

(CC BY 4.0) | ISSN 2525-3409 | DOI: http://dx.doi.org/10.33448/rsd-v10i6.15964

Martins, A. M. F et al. (2020). A Saúde da pessoa idosa no contexto da pandemia pelo coronavírus: Considerações para enfermagem. Revista de enfermagem do Centro-Oeste Mineiro.

Morais, C. L et al. (2020). Violência contra o idoso durante a pandemia de Covid-19 no Brasil: Contribuições para seu enfrentamento. Instituto de Medicina social.

Nabuco, G, M. H. P. P, \& Afonso, M. P. O. (2020). Impacto da pandemia pela COVID-19 na saúde mental: qual é o papel da Atenção Primária à Saúde? Revista Brasileira de medicina de família e comunidade.

Nascimento, J. F. E., Tatmatsu, D. I. B. \& Freitas, R G. T.(2020) Ansiedades em idosos em tempos de isolamento social no Brasil (COVID-19). Revista Brasileira de Análise do Comportamento, 16(1).

Nunes, B. P et al. (2020). Multimorbidade e população em risco para COVID-19 grave no Estudo Longitudinal da Saúde dos Idosos Brasileiros.Caderno de saúde publica.

Raiol, R. A. (2020). Praticar exercícios físicos é fundamental para a saúde física e mental durante a Pandemia da COVID-19. Brazilian Journal of Health Review, 3(2), 2804-2813.

Rocha, S. V. et al. (2020). A pandemia de COVID-19 e a saúde mental de idosos: possibilidades de atividade física por meio dos Exergames. Revista Brasileira de atividade fisica e saúde, Bahia, Brasil.

Santos, J. M. S. Messias, E. M. S., \& Lopes, R..F.(2020). Saúde mental e o isolamento social em períodos de pandemia, Revista Nursing.

Santos, M. F., \& Rodrigues, J. F. S.(2020) COVID-19 e repercussões psicológicas durante a quarentena e o isolamento social: uma revisão integrativa. Nursing, 4095-4100.

Rocha, S. V et al. (2020). A pandemia de COVID-19 e a saúde mental de idosos: possibilidades de atividade física por meio dos Exergames. Revista Brasileira de atividade física e saúde, Bahia, Brasil.

Romero, D. E et al. (2020). Idosos no contexto da pandemia da COVID-19 no Brasil: efeitos nas condições de saúde, renda e trabalho, caderno de saúde pública.

Watanabe, H. A. W., Marisa, A. R. C. D., \& Yeda, A. O. D. (2020) Revista Científica da Sociedade de Geriatria e Gerontologia, 14.

Watanabe, H., Domingues, M., \& Duarte, Y.(2020) COVID-19 e as Instituições de longa permanência para idosos: Cuidado ou morte anunciada. Geriatr Gerontol Aging. 